\title{
Frecuencia de eventos adversos de la terapia endodóntica y seguimiento de pacientes atendidos en el Posgrado de Endodoncia de la Pontificia Universidad Javeriana (2007-2008)
}

\author{
Frequency of Endodontic Treatment Adverse Events and Follow- \\ up of Patients seen at the Endodontics Postdoctoral Clinic of \\ the Pontificia Universidad Javeriana (2007-2008)
}

Marcela Carolina Tafur Gallego Odontóloga, especialista en Endodoncia, Pontificia Universidad Javeriana, Bogotá, Colombia. Práctica privada.

Luis Diego Camacho Alonso Odontólogo, Universidad Latina de Costa Rica, San José, Costa Rica. Especialista en Endodoncia, Pontificia Universidad Javeriana, Bogotá, Colombia.

Santiago Hernando Mejía Morales Odontólogo, especialista en Endodoncia, docente, director del Posgrado en Endodoncia, Pontificia Universidad Javeriana, Bogotá, Colombia.

Juliana González Moncada Odontóloga, directora de Clínicas, Preclínicas y Laboratorios, Pontificia Universidad Javeriana, Bogotá, Colombia. Especialista en Gerencia en Calidad, Universidad El Bosque, Bogotá, Colombia.

María Fernanda Huertas de Hoyos Odontóloga, magistra en Administración en Salud, docente asistente, coordinadora de Clínicas, Pontificia Universidad Javeriana, Bogotá, Colombia.

CÓMO CITAR ESTE ARTíCULO Tafur MC, Camacho LD, Mejía SH, González J, Huertas MF. Frecuencia de eventos adversos de la terapia endodóntica y seguimiento de pacientes atendidos en el Posgrado de Endodoncia de la Pontificia Universidad Javeriana (2007-2008) la Pontificia Universidad Javeriana (2007-2008).
Univ Odontol. 2014 Jul-Dic; 33(71): _. . http:// dx.doi.org/10.11144/Javeriana.uo33-71.feat

doi:10.11144/Javeriana.uo33-71.feat

Recibido para publicación: 05/12/2013 Aceptado para publicación: 24/08/2014

Disponible en: http://www.javeriana.edu.co/ universitasodontologica

\section{RESUMEN}

Antecedentes: los errores clínicos en la prestación de servicios de salud pueden deteriorar más la salud de un paciente. El sistema de salud de países como Colombia establece la obligatoriedad de reportar y hacer seguimiento de tales eventos adversos. Propósito: determinar la frecuencia y la naturaleza de los eventos adversos identificados en las historias clínicas de pacientes que asistieron al Posgrado de Endodoncia de la Pontificia Universidad Javeriana en Bogotá Colombia (2007-2008) y describir el seguimiento realizado a los casos reportados en el primer periodo del 2007. Métodos: en este estudio descriptivo se analizaron 464 historias clínicas en las que se identificaron eventos adversos de tratamientos endodónticos en la hoja de evolución, la secuencia radiográfica y la confirmación en el sistema (modelo se identificación y seguimiento de eventos adversos). Dichos eventos pudieron ocurrir en los procedimientos de apertura, preparación, obturación y después del tratamiento. Resultados: se encontraron 306 eventos adversos en endodoncia (74,4 \%). De ellos, $81,3 \%$ eran eventos prevenibles en procedimientos apertura (4,1\%), preparación $(36,1 \%)$ y obturación $(60 \%)$. Las perforaciones coronales, transportaciones y sobreobturaciones fueron los más frecuentes, respectivamente, en cada tipo de procedimiento. Por otra parte, se identificaron signos de enfermedad en un $26,3 \%$ de los casos de seguimiento analizados del primer periodo del 2007. Conclusión: dada la alta frecuencia de eventos adversos prevenibles en endodoncia identificados en este estudio, es importante generar mecanismos para determinar las causas y generar estrategias para disminuirlas.

\section{PALABRAS CLAVE}

evento adverso; historia clínica; seguridad del paciente; terapia endodóntica

\section{ÁREAS TEMÁTICAS}

Administración en salud; endodoncia

\section{ABSTRACT}

Background: Clinical errors in health care can complicate even more a patient's health. In health care systems such as the Colombian it is compulsory to report and follow up such adverse events. Purpose: Describe the frequency and nature of adverse events identified in the clinical records of patients who attended the Endodontics Postdoctoral Clinics of the Pontificia Universidad Javeriana in Bogotá Colombia (2007-2008) and to describe the follow-up reported cases from the first period of 2007. Methods: In this descriptive study, the follow-up chart, radiographic records, and registry in the system (adverse event identification and follow-up model) of 464 clinical records were analyzed to identify adverse events associated with endodontic therapy. Those events could have happened during root canal opening, clean-up/shaping, filling, and post-treatment. Results: There were 306 adverse events related to endodontic treatment $(74.4 \%) .81 .3 \%$ were preventable events during canal opening (4.1\%), clean-up/shaping (36.1\%), and filling (60\%). Crown perforations, root canal transportation, and overfilling were the most frequent events in the procedures mentioned before. On the other side, there were symptoms reported in $26.3 \%$ of the 2007 first-term follow-up cases studied. Conclusion: Since adverse events in endodontics identified in this study were very frequent, it is important to create mechanisms to determine causes and develop strategies to reduce them.

\section{KEYWORDS}

adverse event; endodontic treatment; medical record; patient safety

THEMATIC FIELDS

Endodontics; health care administration 


\section{INTRODUCCIÓN}

Un evento adverso es el resultado de una atención insegura en salud que, de manera no intencional, produce daño y puede generar un deterioro en la salud de un paciente. Es considerado una lesión atribuible a fallas en la atención y no por la patología de base. Debido a que los eventos adversos ocurren por la práctica clínica, es necesario contar con herramientas y metodologías de gestión para controlarlos de manera oportuna, a fin de someter a un paciente al menor riesgo posible (1-3). Al respecto, la Organización Mundial de la Salud lanzó en el 2005 la Alianza Mundial para la Seguridad Clínica del Paciente, con el objeto de coordinar, difundir y acelerar una mejor calidad en atención. Ya desde 1993, Colombia había iniciado este proceso con la creación del Sistema de Garantía de Calidad en Salud en el marco de la Ley 100 de Seguridad Social Integral, con el propósito de mejorar progresivamente la calidad de la atención en salud.

En la actualidad, la normatividad busca garantizar que se ofrezca un servicio de salud con cubrimiento para toda la población con base en la vigilancia, el reporte y el control de todos los procedimientos, para disminuir al mínimo el riesgo posible de eventos adversos durante la atención clínica en todas las instituciones de salud (1-5). En odontología, la endodoncia es una de las áreas con mayor dificultad de procedimiento, debido a la naturaleza y a la dependencia de factores del huésped previos al tratamiento y a la destreza del operador. En este contexto, la detección y manejo de eventos adversos en endodoncia debe buscar desarrollar herramientas de mejoramiento aplicables a los procesos clínicos, con el fin de limitar su riesgo. Para determinar la conducta de la terapia endodóntica se debe realizar un seguimiento clínico y radiográfico para definir el éxito o el fracaso del tratamiento. De esta manera, se podrá determinar si un evento adverso constituye un daño permanente a una persona (5-7).

Durante mucho tiempo se consideró que los eventos adversos se debían a fallas humanas (fallas activas), resultado de decisiones y acciones del personal que participa en los proceso. Hoy en día, el estudio de los eventos adversos pone el relieve en fallas del sistema, denominadas fallas latentes, y que se asocian a deficiencias en los procesos, el diseño en la organización y la implementación de los productos y sistemas de reporte. El error humano es apenas una parte del problema de fallas en la seguridad del paciente. Hay que tener en cuenta que en el evento adverso el paciente y el profesional sufren daño; por tal razón, es necesa- rio estudiar la cadena de procesos de la atención en salud que, al fallar, facilitaron el desarrollo de dicho evento $(3,7,8)$.

La política de seguridad del paciente es un principio fundamental en el manejo de la calidad. Esta incluye un sistema conceptual y metodológico para implementar sistemáticamente en una institución de salud un conjunto articulado de conceptos, políticas, estrategias y herramientas de amplia acción. Involucra tres acciones complementarias: prevenir eventos adversos, hacerlos visibles y mitigar sus efectos cuando ellos ocurran. Incluye la identificación y el análisis de los eventos adversos, sus causas y las acciones para intervenirlos mediante barreras para cada uno de los eventos identificados (6,8-11).

La seguridad clínica del paciente se ha convertido en motivo de investigación, debido al incremento de eventos adversos, reportados a partir del 2002, en países desarrollados y que se derivan de la práctica clínica en áreas como medicina, enfermería y odontología. Esto indica que existen problemas de atención en salud que llevan al aumento de costos e implicaciones legales, además de un deterioro de la salud del paciente por la presencia de secuelas transitorias o permanentes. Ello insta a la reflexión bioética de la relación profesional de la salud-paciente para la gestión de la calidad asistencial $(5,9,11)$. En el 2004, la Alianza Mundial para la Seguridad Clínica buscó disminuir los riesgos de enfermedad posteriores a la atención en salud, mediante la evaluación de las fases de diagnóstico y terapéutica, con el fin de establecer estrategias de calidad y seguridad por medio de la identificación y vigilancia del evento adverso $(1,2)$. En Colombia, con la Resolución 1446 del 2006, se establece la obligatoriedad de la vigilancia y seguimiento de eventos adversos, cuya finalidad es el aprendizaje para generar barreras de seguridad y no su uso como mecanismo de culpabilización $(6,7,12,13)$. Con el Decreto 2376 del 2010 de Colombia se determinan las normas que reglamentan la relación docenteasistencial, al establecer que:

La estrategia pedagógica planificada y organizada en una institución educativa busca integrar la formación académica con la prestación de servicios de salud, con el propósito de fortalecer y generar competencias, capacidades en los estudiantes de programas de salud en un marco que promueve la calidad de atención y el ejercicio autónomo, responsable y ético. (13-15) 
Con el objetivo de mantener la cultura de reporte y vigilancia de eventos adversos en endodoncia, esta investigación tuvo como fin identificar los eventos adversos más frecuentes reportados en las historias clínicas de los pacientes del Posgrado de Endodoncia de Pontificia Universidad Javeriana de Bogotá. Asimismo, se buscó identificar el seguimiento efectuado a los eventos adversos reportados para ratificar si mostraron signos de enfermedad. Para ello, se empleó un modelo de identificación y seguimiento de eventos adversos desarrollado por el grupo de investigación. El propósito es señalar la implementación de barreras de control y prevención y, de esta manera, contribuir a la seguridad clínica de los pacientes y generar entre el personal prestador de servicios de salud la adopción de prácticas seguras.

\section{MATERIALES Y MÉTODOS}

En este estudio descriptivo se analizaron 411 historias clínicas de pacientes que asistieron al Posgrado de Endodoncia de la Pontificia Universidad Javeriana entre el segundo periodo del 2007 y el segundo periodo del 2008, y a quienes se les realizaron procedimientos de endodoncia convencional. Igualmente, se evaluó el seguimiento registrado en 53 historias clínicas del primer periodo de 2007 y en las que se reportaron eventos adversos en la parte anterior. Las historias clínicas cumplieron con los criterios de inclusión, basados en los hallazgos de las observaciones reportadas en el sistema y que confirmó el docente a cargo con respecto al procedimiento de endodoncia convencional. Asimismo, se requería que se encontraran en el archivo de gestión el día de la búsqueda realizada, que contuvieran letra y radiografías completas y legibles y que la hoja de evolución estuviera firmada por el docente, el estudiante y el paciente. Como criterios de exclusión se consideraron que la hoja de evolución no presentara detalle cronológico del tratamiento o controles realizados y que solo tuvieran radiografías tomadas con sistema digital.

Antes de desarrollar la prueba, se validó el contenido de los formatos de recolección de información, por medio de una prueba piloto basada en la selección de tres endodoncistas, docentes de la Facultad de Odontología de la Pontificia Universidad Javeriana, con conocimientos en los modelos de gestión en seguridad del paciente. Cada uno diligenció el formato de identificación y de seguimiento de eventos adversos en endodoncia. Posteriormente, se aplicó y analizó la encuesta de validación de contenido de los formatos de recolección, con lo cual se realizaron los ajustes pertinentes, según el análisis hecho en la prueba piloto y comenzó la recolección de información. Luego, ayudados por la base de datos obtenida del sistema de información, se procedió a iniciar la observación de nuevos eventos adversos en endodoncia en los procedimientos de apertura, preparación, obturación, control postratamiento, así como la presencia o no de signos clínicos y radiográficos de enfermedad en los casos de seguimiento de evento adverso. Los datos obtenidos se analizaron por medio de estadística descriptiva.

\section{RESULTADOS}

El reporte de eventos adversos relacionados con el tratamiento de endodoncia convencional mostró un total de 306 (74\%), los cuales se reportaron en un $51,9 \%$ en las historias clínicas y se clasificaron por medio de la tabla 1.

TABLA 1

CLASIIICACIÓN DE EFECTOS ADVERSOS EN ENDODONCIA

\begin{tabular}{lll}
\hline Fase de tratamiento & Eventos adversos prevenibles & Eventos adversos no prevenibles \\
\hline Apertura & Fractura coronal & Imposibilidad de localizar conductos \\
& Perforación coronal-cervical-furca & Hipersensibilidad a los anestésicos \\
Preparación biomecánica & Accidente con hipoclorito de sodio & Hipersensibilidad al hipoclorito de \\
& Broncoaspiración de instrumento & sodio \\
& Fractura de instrumento & \\
& Transportación & \\
& Sobreinstrumentación & \\
& Perforación radicular & \\
& Sobrepreparación lateral & \\
& &
\end{tabular}




\begin{tabular}{lll}
\hline Fase de tratamiento & Eventos adversos prevenibles & Eventos adversos no prevenibles \\
\hline Obturación & $\begin{array}{l}\text { Subpreparación } \\
\text { Fractura de instrumento } \\
\text { Fractura radicular } \\
\text { Quemadura de tejidos } \\
\text { Sobreobturación gutapercha } \\
\text { Sobreobturación cemento } \\
\text { Sobrextensión } \\
\text { Condensación deficiente }\end{array}$ & \\
& \\
Postratamiento & $\begin{array}{l}\text { Contaminación de conductos } \\
\text { Fractura radicular vertical }\end{array}$ \\
& $\begin{array}{l}\text { Perdida de obturación temporal } \\
\text { Reabsorción externa } \\
\text { posblanqueamiento } \\
\text { Inflamación intraoral } \\
\text { Inflamación extraoral }\end{array}$ \\
& Dolor moderado \\
& Dolor severo \\
\hline
\end{tabular}

La figura 1 muestra la frecuencia de eventos adversos encontrados. El mayor número correspondió a eventos adversos prevenibles ( 249 casos: $81,3 \%$ ). Aunque no todos eran reportados en la historia clínica, se registraron más los efectos adversos no prevenibles (70,2\%).

FIGURA 1

FRECUENCIA DE EFECTOS ADVERSOS PREVENIBLES Y NO PREVENIBLES

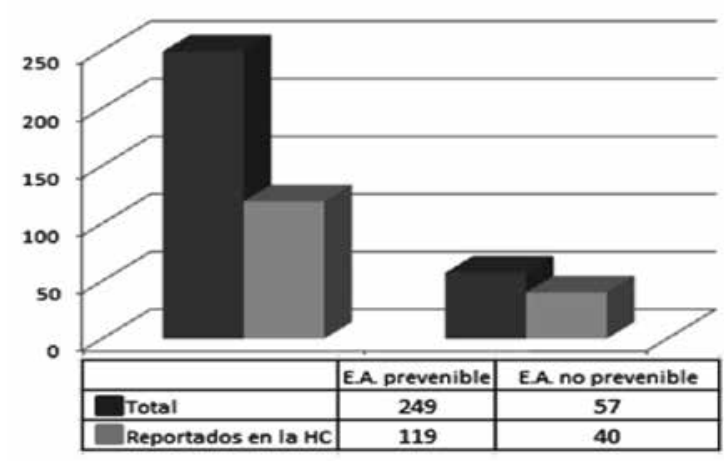

HC: historia clínica.

Entre los eventos adversos prevenibles con respecto al tipo de procedimiento, se observó una alta frecuencia en los procedimientos de preparación (36,1\%) y obturación (59,8\%). No todos los efectos adversos estaban reportados en las historias clínicas. Hubo más paridad entre los efectos adversos prevenibles y no prevenibles en apertura y no prevenibles postratamiento (figura 2).
FIGURA 2

SubCLASIIICACIÓN DE LOS EFECTOS ADVERSOS EN ENDODONCIA
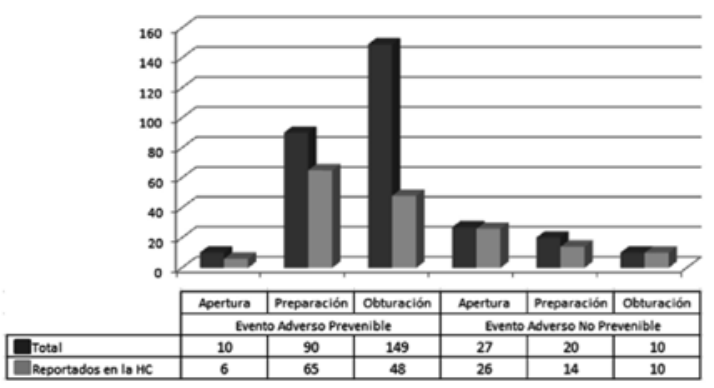

De los 10 casos hallados de eventos adversos prevenibles en apertura ( $4,1 \%$ del total de efectos en apertura), la mayor frecuencia correspondió a perforaciones. Por otra parte, se encontraron 27 casos en los cuales fue imposible localizar conductos, aun con el uso de magnificación y sistemas ultrasónicos. Este último estaba reportado en 26 historias clínicas (figura 3).

FIGURA 3

FRECUENCIA DE EFECTOS ADVERSOS EN APERTURA

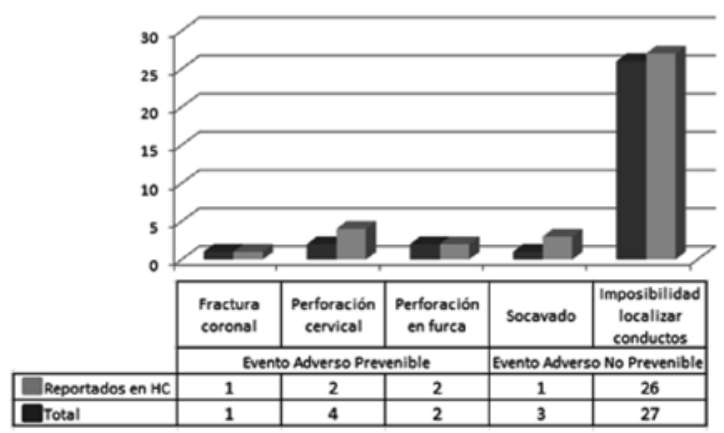


De los 90 (36,1 \% del total) eventos prevenibles durante la preparación biomecánica, se detectó una mayor frecuencia de transportación Este tipo de evento también fue el más reportado en la historia clínica (figura 4). La transportación fue realizada en 31 casos con técnica manual, y en 8 con sistemas rotatorios en dientes multirradiculares. También se identificaron perforaciones radiculares en 26 casos y fractura de instrumentos en 20 casos. Por otro lado, de los 20 eventos adversos no prevenibles, se observó la mayor frecuencia en 14 casos de conductos sin patencia (figura 4).

FIGURA 4

FRECUENCIA DE EFECTOS ADVERSOS EN PREPARACIÓN

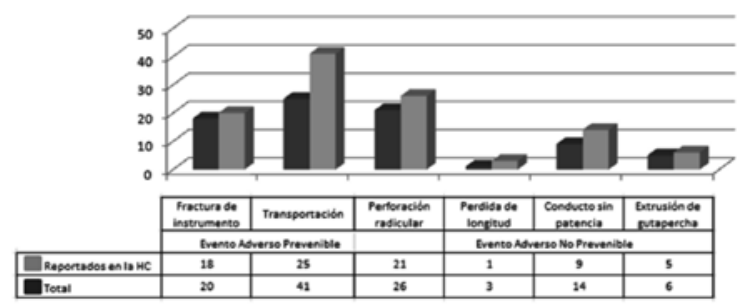

De los 149 casos (60\% del total) de eventos adversos prevenibles en obturación, la mayor frecuencia se halló en sobreobturaciones con cemento y con gutapercha (22 casos). Estaban reportados en la historia clínica 24 casos. Además, se evidenciaron 46 casos de subobturación, 26 de los cuales aparecían reportados en la historia clínica (figura 5). Los eventos adversos no prevenibles ocasionados luego de terminado el tratamiento identificados en el estudio fueron la experiencia de dolor moderado (7 casos) y severo (3 casos). Todos estaban reportados en la historia clínica. La frecuencia observada de estos eventos fue del $17,5 \%$.

FIGURA 5

FRECUENCIA DE EFECTOS ADVERSOS EN OBTURACIÓN

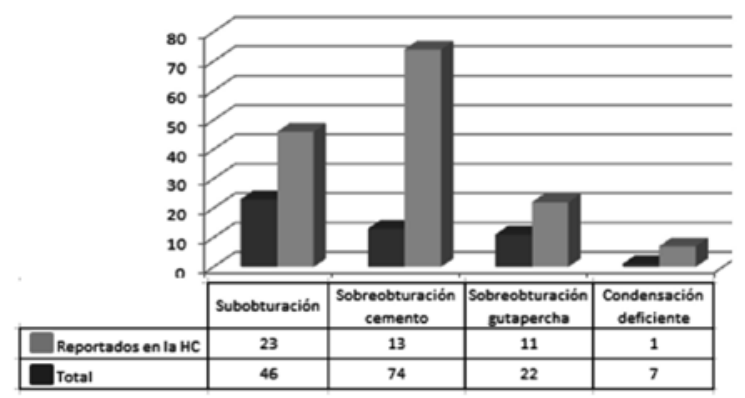

El grupo investigador logró evaluar 53 (51,4 \%) de historias clínicas de seguimiento con reportes de eventos adversos prevenibles y no prevenibles en los procedimientos de apertura, preparación y obturación. En apertura se encontró un total de 13 casos (24,5\%) de los cuales solo 2 (3,7 \%) tuvieron controles, que correspondían a perforación (figura 6).

FIGURA 6

FRECUENCIA DE SEGUIMIENTO DE EFECTOS ADVERSOS EN APERTURA

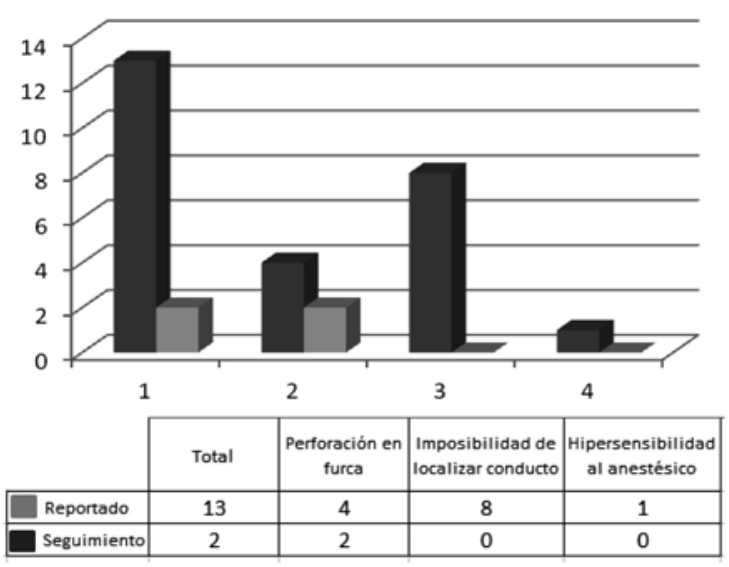

Con respecto al seguimiento de eventos adversos prevenibles y no prevenibles en preparación, se observó que del total de 40 casos hallados (75,4\%), solo 26 (49\%) presentaban controles. Entre ellos, la mayor frecuencia de seguimiento se presentó en los casos de perforaciones radiculares (100\%), transportaciones $(20,7 \%)$ y fractura de instrumento (15\%) (figura 7$)$.

FIGURA 7

FRECUENCIA DE SEGUIMIENTO DE EFECTOS ADVERSOS EN PREPARACIÓN

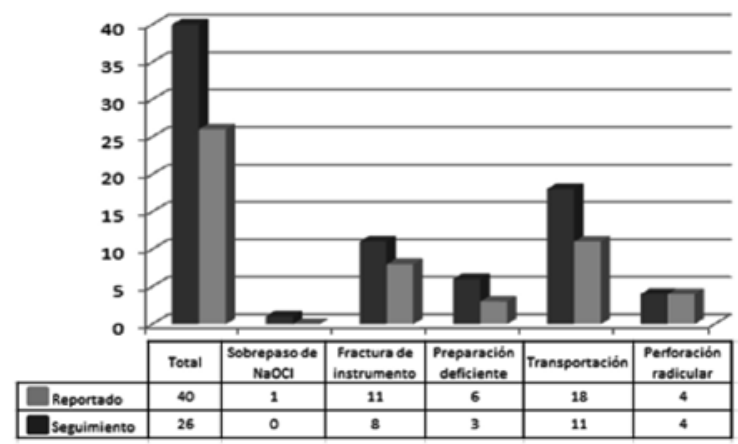

Ahora bien, de los 50 casos observados (48,5\%) de seguimiento de eventos adversos en obturación, 25 casos (47 \%) tenían controles. Ellos correspondían a subobturación (37,7 \%) y sobreobturación (100 \%) (figura 8). 
FIGURA 8

FRECUENCIA DE SEGUIMIENTO DE EVENTOS ADVERSOS EN OBTURACIÓN

El único caso de seguimiento de evento adverso no prevenible postratamiento identificado fue una pérdida de obturación coronal. Se describe en la evolución de la historia clínica que el paciente asistió a los 8 meses de terminado el tratamiento con una lesión clínica compatible con fisura coronal. Radiográficamente, se observaba una lesión lateral, cuyo tratamiento fue exodoncia.

La frecuencia total de eventos adversos prevenibles en endodoncia durante apertura, preparación y obturación fue 13 casos (24,5\%). Se observó transportación, en el 30,7 \% de los casos; perforación, en el 23 $\%$, y fractura de instrumento, en el $15,3 \%$. Por otro lado, los eventos adversos no prevenibles se encontraron en un $1,8 \%$. Cabe anotar que entre los signos radiográficos de enfermedad, el más observado fue la lesión apical, en un 71,4 \% de los casos (tabla 2).

\section{DISCUSIÓN}

En el 2008, el Posgrado de Endodoncia de la Pontificia Universidad Javeriana de Bogotá inició el proceso de reconocer los eventos adversos ocurridos durante la atención clínica, con el fin de evitar su aparición y establecer un modelo de control y seguimiento de los casos identificados (15). Continuando con esta línea de investigación, el presente estudio se realizó para determinar la frecuencia y naturaleza de los eventos adversos detectados en las historias clínicas de los pacientes que asistieron a las clínicas del posgrado entre el segundo periodo del 2007 y el segundo periodo del 2008 mediante el análisis de reportes, así como el seguimiento de los casos reportados del primer periodo del 2007.

En la literatura se encuentran pocos estudios acerca de los eventos adversos ocurridos durante la terapia endodóntica convencional o después de esta. Aquí se encontraron eventos adversos en un $74,4 \%$ de las historias clínicas revisadas. Ello es similar a lo hallado por Selbst (16) y Giorgos y colaboradores (17), quienes reportaron un 63,6 \% de casos con eventos adversos durante procedimientos de endodoncia convencional. Esta investigación subclasificó los eventos adversos prevenibles y no prevenibles en las diferentes fases del procedimiento endodóntico y encontró frecuencias del 4,1 \% y 47,3\%, respectivamente, en procedimientos de apertura que estuvieron relacionados con fractura coronal, socavados,

TABLA 2

EFECTOS ADVERSOS CON SIGNOS DE ENFERMEDAD Y TRATAMIENTO REALIZADO

\begin{tabular}{lclll}
\hline Evento adverso & Total con signos & Signo radiográfico & Signo clínico & Tratamiento \\
\hline Perforación furca & 1 & Lesión furca & $\begin{array}{l}\text { Dolor: } 1 \\
\text { No reporta: } 1\end{array}$ & Exodoncia \\
$\begin{array}{lcll}\text { Fractura de } \\
\text { instrumento }\end{array}$ & Lesión apical: 2 & $\begin{array}{l}\text { Dolor: } 1 \\
\text { No reporta: } 2\end{array}$ & Cirugía apical: 2 \\
\hline $\begin{array}{l}\text { Perforación radicular } \\
\text { Transportación }\end{array}$ & 3 & Lesión apical & Dolor & Exodoncia \\
& 4 & No reparación & Movilidad & No reporta \\
& & Lesión apical & No reporta & Amputación \\
& & Lesión apical & Dolor & Cirugía apical \\
& & Lesión apical & No reporta & Cirugía apical \\
Lesión apical & No reporta & Cirugía apical \\
Cirugía apical
\end{tabular}


perforaciones cervical y en furca, e imposibilidad de localizar conductos.

Como se observa en la figura 3, las perforaciones se presentaron en mayor proporción que los demás eventos (2,4\%). Hernández y colaboradores (15) reportaron un $1,12 \%$ de dichos eventos. Selbst (16) informa que la incidencia de perforaciones se encuentra en un rango entre $2 \%$ y $12 \%$. Las perforaciones y socavados se pueden presentar por calcificaciones, cámaras pulpares retraídas, dientes en malposición, presión excesiva y alineamiento de las fresas en eje del diente, lo que podría ocasionar desviaciones durante el proceso de apertura, debilitamiento excesivo de la estructura dental remanente comprometiendo el pronóstico dental e impidiendo su posterior rehabilitación. Las perforaciones en el área de la furca provocan inflamación inmediata de la zona, más aún si existe comunicación con el medio oral. Esto se puede prevenir mediante el aislamiento del campo absoluto; aun así, esta situación puede predisponer al desarrollo de infección y lesión periodontal, ya que la relación con la cresta ósea y el epitelio de unión del surco gingival favorece la contaminación con bacterias de la cavidad oral. Ello disminuye el potencial de reparación y puede permitir la proliferación epitelial haciendo el pronóstico dudoso, así como la afección del hueso alveolar que puede llevar rápidamente a la pérdida del diente involucrado. En el seguimiento se ratificó que este tipo de eventos ocasiona signos de enfermedad, riesgo que debe ser advertido al paciente (tabla 1) (16-20).

La posibilidad de localización de conductos radiculares calcificados bajo el ojo humano es solamente de un $17 \%$, lo cual es relativamente inefectivo si se compara con el $80 \%$ de efectividad con el uso de microscopio. La imposibilidad de localizar conductos puede originar un fracaso endodóntico debido a que estos, como se ha demostrado histológicamente, a pesar de no ser visibles clínica o radiográficamente, nunca se calcifican en su totalidad. Por eso, en el conducto puede haber tejido inflamatorio o necrótico que, al no poder ser instrumentado, puede hacer que persista una infección y genere fracaso. En estos casos se debe explicar al paciente el riesgo-beneficio de una búsqueda exhaustiva de un conducto, por medio de tecnología, como magnificación y ultrasonido. El progreso se debe comunicar al paciente durante el procedimiento y reportarlo en la historia clínica y registrarlo en el sistema $(21,22)$.

Con respecto a los eventos adversos prevenibles durante la fase de preparación, en este estudio se en- contró que las transportaciones se presentaron con una frecuencia del 16,4%. El grupo de Hernández y colaboradores, de la misma institución, reportó una frecuencia de este tipo de evento en un 15,9 \% (15). En la literatura se reporta una incidencia de entre el 23 $\%$ y el $68 \%$ de transportaciones en estudios in vitro, que es mayor en dientes curvos preparados con técnicas manuales y limas de acero inoxidable. Las limas dejan superficies irregulares que impiden la completa limpieza biomecánica, lo que produce una irritación crónica del tejido periapical asociada a factores como bacterias, tejido necrótico y dentina contaminada, y hace necesario emplear terapias quirúrgicas. Con respecto a los casos de seguimiento, se confirmó que la transportación ocasionó signos de enfermedad (tabla 2). Con el fin de disminuir las transportaciones, se ha sugerido el uso de instrumentos de níquel-titanio, por sus características de elasticidad y memoria de forma, lo que permite manejar mejor los dientes con curvaturas. Sin embargo, como se identificó en esta investigación, la fractura de instrumentos se observó en un $8 \%$, con un 5,2\% por instrumentos rotatorios. En el estudio de Hernández y colaboradores se había reportado un 3,9\% para este tipo de evento (15). La literatura reporta una frecuencia de entre el 1,3\% y el $10 \%$. Este rango puede ser el resultado del uso inadecuado, fuerza excesiva o falta de entrenamiento del operador. Por lo tanto, es posible que este tipo de error esté asociado con deficiencias en el proceso de enseñanza-aprendizaje (23-25).

En la literatura se reporta que el instrumento fracturado no es el responsable directo del fracaso endodóntico; no obstante, impide la limpieza del conducto radicular donde la infección se perpetúa y es causa primaria del fracaso. Por otro lado, si el instrumento queda localizado más allá de la longitud de trabajo, puede generar una reacción a cuerpo extraño, debido a la irritación constante del tejido periapical. Asimismo, intentar efectuar una preparación correcta puede llevar a perforaciones, escalones o transportaciones. Los casos así deben ser controlados, y si los síntomas persisten, es fundamental considerar terapias quirúrgicas. Entre los casos de seguimiento, se confirmó que este evento puede llegar a ocasionar signos de enfermedad (tabla 2) (26-29).

En cuanto a las perforaciones radiculares, en este estudio se encontraron en un 10,4\%; mientras que el grupo de Hernández y colaboradores se observó un $1,12 \%$ (15). En la literatura se reporta una frecuencia del 2,7\% al $10 \%$. Estos hallazgos indican que el clínico debe ser más cuidadoso para evitar esta 
clase de eventos adversos. El pronóstico de un diente tratado endodónticamente con una perforación es incierto y depende de muchos factores, como el lapso que transcurre entre el accidente y la perforación, el material de sellado del defecto y el tamaño de la perforación. En cuanto al tiempo, se reporta que es mucho mejor cuando se produce el sellado de forma inmediata. Cualquier retardo en el selle de la perforación, aun bajo condiciones de asepsia, puede resultar en infección y retardar el proceso de reparación. Si la perforación es expuesta al medio oral y no se sella de manera inmediata, el pronóstico es desfavorable (1820). Con respecto a las perforaciones pequeñas, hay una asociación entre el menor daño al tejido periodontal y cambios patológicos (46\%). A mayor tamaño, aumenta el potencial de riesgo de sobreobturación y sellado inadecuado, ya que existe la probabilidad de daño periodontal en un 80 \% (16). Muchos autores coinciden en que el pronóstico de una perforación que ocurre en el tercio medio o el apical es mejor que la perforación en el tercio cervical o en la furca. Por otro lado, de los casos de perforación con seguimiento, se observó que hubo una lesión al paciente (tabla 2) $(18,19)$.

La literatura reporta entre un 12,6\% y un 21,6\% de sobreobturaciones. En esta investigación se encontraron sobreobturaciones en un $29,7 \%$ y un $8,8 \%$ con cemento y gutapercha, respectivamente. En el estudio de Hernández y colaboradores (15) únicamente se reportó un 1,7 \%. De acuerdo con el porcentaje observado, se debe buscar la forma de evitar este tipo de situaciones. Se ha reportado que la sobreobturación con cemento sellador ocasiona una respuesta inflamatoria y retardo en la reparación, debido a la citotoxicidad de los cementos. Asimismo, la extrusión de gutapercha induce reacción inflamatoria, lo que ocasiona reacción por cuerpo extraño con dolor o persistencia de los síntomas (30-37). De acuerdo con estas observaciones, los casos de sobreobturación deben estar bajo un estricto control para así descartar algún signo de enfermedad en el paciente. Esto se plantea porque en la literatura se ha reportado que dentro de las posibles causas de fracasos endodónticos hay factores no microbiológicos, como cuando hay sobreobturación con materiales de obturación endodónticos $(30,38)$.

Los estudios reportan que la subobturación se encuentra en una frecuencia del $25 \%$. En el presente estudio se halló en un 18,4 \% de los casos; mientras que Hernández y colaboradores hallaron una frecuencia del 11,9 \% con 3 casos que presentaron signos de enfermedad (tabla 2). La subobturación impide una adecuada instrumentación por una longitud de trabajo inexacta, la cual ocurre como resultado de un evento adverso previo como escalones, transportaciones o bloqueo. Esto puede incidir en la persistencia o la aparición de lesión apical (22,35,37).

Otro aspecto relevante identificado en el presente estudio es que solo un $47,8 \%$ de los casos con evento adverso prevenible estaban reportados en la historia clínica, lo cual indica una carencia en la cultura del reporte. Asimismo, 28 casos de reportes de seguimiento de eventos adversos no tenían controles y presentaban deficiencias en el diligenciamiento de la evolución y examen radiográfico, que debían ser efectuados según el protocolo de la clínica del Posgrado de Endodoncia. Al considerar que los hallazgos de eventos adversos prevenibles son un resultado no deseado, causado sin intención, es importante pensar que se pueden evitar mediante el correcto cumplimiento de los estándares del cuidado asistencial. Es importante dar información oportuna al paciente del evento ocasionado, una mayor atención del caso y una evolución detallada en la historia clínica.

\section{CONCLUSIÓN}

La utilización de herramientas de gestión clínica como un modelo de identificación y seguimiento de eventos adversos contribuyó a conocer la frecuencia de eventos adversos en endodoncia. Ello ayuda a generar una alerta en la institución que permita conocer las causas de las fallas humanas y del sistema durante los procedimientos, para crear las barreras de control necesarias, disminuir esta frecuencia y generar una atención de calidad y seguridad al paciente. Del mismo modo, una vez identificados los eventos adversos, deben ser estrictamente seguidos dentro del Posgrado de Endodoncia y reportados de manera detallada en la historia clínica y confirmado adecuadamente en el sistema por parte del docente a cargo.

\section{RECOMENDACIONES}

Generar una base de datos con información de contacto de pacientes para poder citarlos a controles en la Facultad de Odontología y que sean atendidos por los residentes del Posgrado de Endodoncia, a fin de realizar un estricto seguimiento y vigilancia por parte del docente. Se debe también determinar el riesgo de daño al paciente por los eventos adversos identificados y, del mismo modo, proporcionar la terapéutica adicional requerida para mantener la salud oral. 
Seguir fomentando la cultura del reporte del evento adverso en el ámbito universitario como mecanismo de mejoramiento y prevención en el sistema de calidad de salud.

Inculcar en el paciente la necesidad de asistir a las citas de control para conocer los resultados del tratamiento realizado.

Incluir un ítem de eventos adversos en endodoncia, donde se pueda diligenciar de manera precisa y fácil el evento y tener un control estricto de los casos reportados por parte del área administrativa.

Actualizar los protocolos de seguimiento de riesgo en endodoncia de acuerdo con los eventos adversos reportados y hacer partícipes de estos a los estudiantes del posgrado.

\section{AGRADECIMIENTOS}

A las doctoras Adriana Rodríguez y Gloria Moreno, por su valiosa colaboración en la orientación metodológica de este trabajo.

\section{REFERENCIAS}

1. Franco A. Fundamentos de seguridad al paciente para disminuir errores médicos. 1a ed. Cali, Colombia: Editorial Universidad del Valle; 2006.

2. Aguirre-Gas HG, Vázquez-Estupiñán F. Error médico: eventos adversos. Cirugía y Cirujanos. 2006; 74(6): 495503.

3. Franco A. El reporte de eventos adversos: motor de gestión clínica. Vía Salud. 2005 Oct-Dic; (38): 10-4.

4. Organización Mundial de la Salud. Memorias de la 55a Asamblea Mundial de la Salud. Ginebra, Suiza; 2002.

5. Organización Mundial de la Salud. International classification for patient safety. Geneva, Switzerland; 2007.

6. Franco A. La seguridad clínica de los pacientes: entendiendo el problema. Colomb Med. 2005 Abr-Jun; 36(2): 130-3.

7. Mejía C, Portocarrero J. Gestión clínica, eventos adversos y responsabilidad civil médica. Vía Salud. 2006 Abr-Jun; (32): 10-3.

8. Blanco S, Carrero D, Mejía A, Moreno A, Ramírez D, Ramírez R, Virgüez O. La oficina de seguridad del paciente como estrategia integral de gestión de riesgos en un servicio ambulatorio. Vía Salud. 2012 Jul-Sep; 16(2): 6-10.

9. Ospina J. Seguridad del paciente en atención primaria. Vía Salud. 2011 Oct-Dic; (58): 18-25.

10. Dirección General de Calidad de Servicios. Guía Técnica "Buenas prácticas para la seguridad del paciente en la atención en salud”. Bogotá: Ministerio de Protección Social, Centro de Seguridad del Paciente; 2008.

11. Portocarrero J. Actividades y experiencias en materia de calidad de los servicios de salud en Colombia. Vía Salud. 2009 Dic; (50): 4-10.

12. Kerguelén C. Reporte y análisis de eventos adversos: la necesidad de estructurar sus relaciones. Vía Salud. 2010 Jul; (52): 3-11.

13. Villarreal Cantillo E. Seguridad de los pacientes: Un compromiso de todos para un cuidado de calidad. Vía Salud. 2007; 23(1): 112-9.

14. Quintero Hernández G. Gestión de la curva de aprendizaje. Vía Salud. 2010; (56); 12-20.

15. Hernández S, Laiseca N, Rincón A. Identificación de eventos adversos que se presentaron en los pacientes que asistieron al posgrado de endodoncia de la Pontificia Universidad Javeriana en el primer periodo del año 2007. Trabajo de posgrado en Endodoncia. Bogotá, Colombia: Pontificia Universidad Javeriana; 2008.

16. Selbst AG. Understanding informed consent and its relationship to the incidence of adverse treatment events in conventional endodontic therapy. J Endod. 1990 Aug; 16(8): 387-90.

17. Giorgos N, Tzanetakis GN, Evangelos G, Kontakiotis, Dimitra V. Prevalence and management of instrument fracture in the postgraduate endodontic program at the Dental School of Athens: a five- year retrospective clinical study. J Endod. 2008 Jun; 34(6): 675-8.

18. Suter B, Lussi A, Sequeira P. Probability of removing fractured instruments from root canals. J Endod. 1990 Aug; 16(8): 387-90.

19. Morse DR. Age-related changes of the dental pulp complex and their relationship to systemic aging. Oral Surg Oral Med Oral Pathol. 1991 Dec; 72(6): 721-45.

20. Sener S, Cobankara FK, Akgünlü F. Calcifications of the pulp chamber: Prevalence and implicated factors. Clin Oral Investig. 2009 Jun; 13(2): 209-15.

21. Yildirim G, Dalci K. Treatment of lateral root perforation with mineral trioxide aggregate: a case report. Oral Surg Oral Med Oral Pathol Oral Radiol Endod. 2006 Nov; 102(5): e55-8.

22. Tsatsas DV, Meliou HA, Kerezoudis NP. Sealing effectiveness of materials used in furcation perforation in vitro. Int Dent J. 2005 Jun; 55(3): 133-41.

23. Sonntag D, Stachniss-Carp S, Stachniss C, Stachniss V. Determination of root canal curvatures before and after canal preparation (part II): a method based on numeric calculus. Aust Endod J. 2006 Apr; 32(1): 16-25.

24. Kfir A, Rosenberg E, Zuckerman O, Tamse A, Fuss Z. Comparison of procedural errors resulting during root canal preparations completed by senior dental students in patients using an "8-step method" versus "serial stepback technique”. Oral Surg Oral Med Oral Pathol Oral Radiol Endod. 2004 Jun; 97(6): 745-8.

25. Gutmann JL, Leonard JE. Problem solving in endodontic working-length determination. Compend Contin Educ Dent. 1995 Mar; 16(3): 288, 290, 293-4, 304. 
26. Pettiette MT, Metzger Z, Phillips C, Trope M. Endodontic complications of root canal therapy performed by dental students with stainless-steel K-files and nickel-titanium hand files. J Endod. 1999 Apr; 25(4): 230-4.

27. Martín B, Zelada G, Varela P, Bahillo JG, Magán F, Ahn S, Rodríguez C. Factors influencing the fracture of nickeltitanium rotary instruments. Int Endod J. 2003 Apr; 36(4): 262-6.

28. Ankrum MT, Hartwell GR, Truitt JE. K3 Endo, ProTaper, and ProFile systems: breakage and distortion in severely curved roots of molars. J Endod. 2004 Apr; 30(4): 234-7.

29. Lam TV, Lewis DJ, Atkins DR, Macfarlane RH, Clarkson RM, Whitehead MG, Brockhurst PJ, Moule AJ. Changes in root canal morphology in simulated curved canals over-instrumented with a variety of stainless steel and nickel titanium files. Oral Surg Oral Med Oral Pathol Oral Radiol Endod. 2006 Nov; 102(5): e55-8.

30. Genet JM, Hart AA, Wesselink PR, Thoden van Velzen SK. Preoperative and operative factors associated with pain after the first endodontic visit. Int Endod J. 1987 Mar; 20(2): 53-64.

31. Willershausen B, Marroquín BB, Schäfer D, Schulze R. Cytotoxicity of root canal filling materials to three different human cell lines. J Endod. 2000 Dec; 26(12): 703-7.

32. Miletić I, Anić I, Karlović Z, Marsan T, Pezelj-Ribarić S, Osmak M. Cytotoxic effect of four root filling materials. Endod Dent Traumatol. 2000 Dec; 16(6): 287-90.

33. Huang FM, Tai KW, Chou MY, Chang YC. Cytotoxicity of resin-, zinc oxide-eugenol-, and calcium hydroxide-based root canal sealers on human periodontal ligament cells and permanent V79 cells. Int Endod J. 2002 Feb; 35(2): 153-8.

34. Heidari M, Bahrami ZS, Shokri F. In vitro cytotoxicity of a new epoxy resin root canal sealer. J Endod. 2000 Aug; 26(8): 462-5.

35. Lin LM, Rosenberg PA, Lin J. Do procedural errors cause endodontic treatment failure? J Am Dent Assoc. 2005 Feb; 136(2): 187-93, 231.

36. Eleftheriadis GI, Lambrianidis TP. Technical quality of root canal treatment and detection of iatrogenic errors in an undergraduate dental clinic. Int Endod J. 2005 Oct; 38(10): 725-34.

37. Chen SC, Lee CM, Hsu YY, Pai SF, Kuo ML, Chen CS, Duh BR, Yang SF, Tung YL, Hsiao CK. Technical quality of root canal treatment in Taiwan. Int Endod J. 2003 Jun; 36(6): 416-22.

38. Siqueira JF Jr. Aetiology of root canal treatment failure: why well-treated teeth can fail. Int Endod J. 2001 Jan; 34(1): 1-10.

\section{CORRESPONDENCIA}

Marcela Carolina Tafur Gallego

marcelatafur24@hotmail.com

Luis Diego Camacho Alonso

camacholuisdiego@hotmail.com
Santiago Hernando Mejía Morales

mejiasantiagosantiago@javeriana.edu.co

Juliana González Moncada

juliana.gonzalez@javeriana.edu.co.

María Fernanda Huertas de Hoyos huertasm@javeriana.edu.co. 